\title{
4CPS-229 CLINICAL MANAGEMENT OF MALIGNANT MESOTHELIOMA IN AN ASBESTOS-ENDEMIC AREA
}

'M Hijazi Vega*, ${ }^{1}$ P López Méndez, 'L López Esteban, ${ }^{1} R$ Diez Fernández, ${ }^{2} A M$ Sánchez Peña, ${ }^{1} T$ Molina García. 'Hospital Universitario de Getafe Madrid, Pharmacy, Getafe, Spain; ${ }^{2}$ Hospital Universitario de Getafe Madrid, Oncology, Getafe, Spain

10.1136/ejhpharm-2019-eahpconf.378

Background Malignant mesothelioma (MM) is a rare cancer, considered an occupational disease in many patients. Incidence is increasing worldwide mainly because of professional exposure to asbestos. MM patients have limited therapeutic options with poor outcomes. Chemotherapy is still the best therapeutic approach.

Purpose We aimed to describe MM patients in an asbestosendemic area and the treatment received since diagnosis. We also aimed to assess treatment efficacy end-points (time to next treatment (TTNT), and progression-free survival (PFS)).

Material and methods Retrospective, descriptive study in a single institution. All MM patients treated with chemotherapy from January 2001 to September 2018 were included. Previous asbestos exposure, radiotherapy, surgery, chemotherapy received and the dates of administration were collected, as well as the dates of the events (change of therapy, radiologic or clinical progression).

Results Fifty-one patients were included ( $84 \%$ males): one was not included in the analysis, as there were no medical records. Median age at initiation therapy was $72.3 \quad(\mathrm{IQR}=6.4)$ years. Eighty-four per cent of patients had previous asbestos exposure; $8 \%$ of patients had pleurectomy or extrapleural pneumonectomy surgery; and $44 \%$ had radiotherapy for pain control. All patients received pemetrexed as first-line treatment $(76 \%$ as a platinum doublet). Half of the patients received secondand subsequent lines of chemotherapy (second-line 44\%, thirdline 24\%; fourth-line 16\%). Drugs used were raltitrexed, gemcitabine, irinotecan and vinorelbine alone or combined. Median TTNT for first-line was $4.2(\mathrm{IQR}=8.8)$; for secondline $2.6(\mathrm{IQR}=2.1)$ and for third-line $2.6(\mathrm{IQR}=4)$ months. Median PFS was $4.5(\mathrm{IQR}=8.1)$ for first-line; $2.3(\mathrm{IQR}=1.6)$ for second-line; 2.7 (IQR=3.6) for third-line; 2.5 $(\mathrm{IQR}=2.8)$ months for fourth-line.

Conclusion Most patients had previous exposure to asbestos. All patients received pemetrexed in the first line of chemotherapy and mostly combined with platinum. Surgery is an option for just a few patients. Radiotherapy is still necessary in many patients for control of symptoms.

As expected, TTNT and PFS diminished with each subsequent chemotherapy line.

\section{REFERENCES AND/OR ACKNOWLEDGEMENTS}

No conflict of interest. 